\title{
The $\sigma$ Subunit-Remodeling Factors: An Emerging Paradigms of Transcription Regulation
}

\author{
Rishi Kishore Vishwakarma* and Konstantin Brodolin* \\ Institut de Recherche en Infectiologie de Montpellier, CNRS, Université de Montpellier, Montpellier, France
}

Transcription initiation is a key checkpoint and highly regulated step of gene expression.

OPEN ACCESS

Edited by: Miroslav Patek, Academy of Sciences of the Czech Republic, Czechia

Reviewed by:

Deborah M. Hinton, National Institutes of Health (NIH),

United States

Fabian Blombach

University College London,

United Kingdom

*Correspondence:

Rishi Kishore Vishwakarma rishivish@gmail.com

Konstantin Brodolin konstantin.brodolin@inserm.fr; konstantin.brodolin@irim.cnrs.fr

tPresent address:

Rishi Kishore Vishwakarma,

Department of Biochemistry and Molecular Biology, The Pennsylvania State University, University Park, PA, United States

Specialty section:

This article was submitted to Microbial Physiology and Metabolism, a section of the journal Frontiers in Microbiology

Received: 30 April 2020 Accepted: 09 July 2020

Published: 29 July 2020

Citation:

Vishwakarma RK and Brodolin $K$ (2020) The $\sigma$ Subunit-Remodeling Factors: An Emerging Paradigms

of Transcription Regulation.

Front. Microbiol. 11:1798.

doi: 10.3389/fmich.2020.01798
The sigma $(\sigma)$ subunit of RNA polymerase (RNAP) controls all transcription initiation steps, from recognition of the $-10 /-35$ promoter elements, upon formation of the closed promoter complex (RPc), to stabilization of the open promoter complex (RPo) and stimulation of the primary steps in RNA synthesis. The canonical mechanism to regulate $\sigma$ activity upon transcription initiation relies on activators that recognize specific DNA motifs and recruit RNAP to promoters. This mini-review describes an emerging group of transcriptional regulators that form a complex with $\sigma$ or/and RNAP prior to promoter binding, remodel the $\sigma$ subunit conformation, and thus modify RNAP activity. Such strategy is widely used by bacteriophages to appropriate the host RNAP. Recent findings on RNAP-binding protein A (RbpA) from Mycobacterium tuberculosis and Crl from Escherichia coli suggest that activator-driven changes in $\sigma$ conformation can be a widespread regulatory mechanism in bacteria.

Keywords: RNAP-binding transcriptional regulators, sigma subunit conformational dynamics, promoter specificity, RbpA, Tuberculosis

\section{INTRODUCTION}

Transcription initiation starts with the assembly of the active RNA polymerase (RNAP) holoenzyme from the catalytic core (subunits $\left.2 \alpha, \beta, \beta^{\prime}, \omega\right)$ and the promoter-specificity subunit sigma $(\sigma)$. The RNAP holoenzyme binds to promoter DNA, and forms a closed promoter complex (RPc) that isomerizes into the open promoter complex (RPo) through several intermediates (Buc and McClure, 1985; Saecker et al., 2011; Boyaci et al., 2019). Upon isomerization, RNAP melts 13 bp of DNA duplex between the promoter positions -11 to +2 that encompass the transcription start site (Figure 1A). Recognition of the -10 (Escherichia coli consensus motif $\mathrm{T}_{-12} \mathrm{~A}_{-11} \mathrm{~T}_{-10} \mathrm{~A}_{-9} \mathrm{~A}_{-8} \mathrm{~T}_{-7}$ ) and -35 elements (E. coli consensus motif $\mathrm{T}_{-35} \mathrm{~T}_{-34} \mathrm{G}_{-33} \mathrm{~A}_{-32} \mathrm{C}_{-31} \mathrm{~A}_{-30}$ ) of the promoter (Figure 1B) and promoter DNA melting depend on the $\sigma$ subunit (Feklistov et al., 2014; Zuo and Steitz, 2015). All bacteria have at least one principal $\sigma$ subunit [group 1: $\sigma^{70}$ in $E$. coli and $\sigma^{\mathrm{A}}$ in other species (Gruber and Gross, 2003)] that ensures transcription of most genes [e.g., at least $70 \%$ in Mycobacterium tuberculosis (Mtb)] (Cortes et al., 2014). Alternative $\sigma$ subunits (groups 2-4) control transcription of specialized sets of genes upon stress response, starvation, and stationary growth (Österberg et al., 2011). Compared with group $1 \sigma$ subunits, the group 2 stress-response/stationary phase $\sigma$ subunits $\left(E\right.$. coli $\sigma^{\mathrm{S}}$ and $M t b \sigma^{\mathrm{B}}$ ) lack the $\mathrm{N}$-terminal variable domain $\sigma 1.1$ and have a shorter non-conserved region (NCR) located in domain $\sigma 2$ (Figure 1C). RNAPs harboring group 1 and group $2 \sigma$ subunits can transcribe the same promoter sets (Rodrigue et al., 2006; Hu et al., 2014). 
The $\sigma 2$ domain harbors the highly conserved regions 1.2, 2.1, $2.2,2.3$, and 2.4 that are essential for binding to RNAP $\beta^{\prime}$ clamp, for recognition of the -10 element, and for melting of promoter DNA (Feklistov and Darst, 2011; Zhang et al., 2012). About $73 \%$ of the $\sigma 2$ contact surface with ssDNA of the -10 element is formed by region 2.3 residues. In addition, residues in region 1.2 contact with the $\mathrm{T}_{-7}$ base of the -10 element (Feklistov and Darst, 2011) and control recognition of the -10 element allosterically (Zenkin et al., 2007; Morichaud et al., 2016). The $\sigma^{70}$ NCR interacts with promoter DNA at positions -16/$17\left(\mathrm{R} 157^{\mathrm{Eco}}\right)$ and is implicated in DNA unwinding (Narayanan et al., 2018). The $\sigma^{70} \mathrm{NCR} / \beta^{\prime}$ interaction facilitates promoter escape (Leibman and Hochschild, 2007). Domain $\sigma 4$ interacts with the $\beta$-flap domain of core RNAP and harbors a helixturn-helix DNA binding domain that recognizes the -35 motif. The $\sigma 3$ and $\sigma 4$ subunits are connected by a weakly structured linker (region 3.2) that fills the RNA exit channel and is ejected upon the initial RNA synthesis (Zhang et al., 2012; Li et al., 2020). Most bacterial promoters recognized by group 1 and $2 \sigma$ subunits belong to the $-10 /-35$ class and contain the -10 and also the -35 elements. The extended -10 class of promoters $(\sim 20 \%$ in $E$. coli) contains the extended -10 motif $\left(\mathrm{T}_{-17} \mathrm{R}_{-16} \mathrm{~T}_{-15} \mathrm{G}_{-14} ; \mathrm{R}=\right.$ purine$)$ that is located one base upstream of the -10 element (Keilty and Rosenberg, 1987; Burr et al., 2000; Mitchell et al., 2003) and interacts with the $\sigma 3$ domain (Barne et al., 1997) (Figure 1B). It has been shown that the extended -10 motif bypasses the requirement of the $\sigma 4 /-35$ element interaction (Kumar et al., 1993). However, $\sigma 4$ per se is essential for transcription initiation by $\sigma^{\mathrm{B}}-M t b \mathrm{RNAP}$ at the extended -10 promoters (Perumal et al., 2018).

As a general rule, the principal $\sigma$ subunit, the cellular concentration of which exceeds that of core RNAP (Gaal et al., 2006) should recognize and bind to promoter DNA only in the context of RNAP holoenzyme. Free $\sigma$ should be devoid of DNA binding activity that might inhibit transcription. Data from structural and biophysical studies suggest that free group 1 and $2 \sigma$ subunits adopt a "closed" inactive conformation in which the spatial arrangement of domains $\sigma 2$ and $\sigma 4$ is incompatible with promoter DNA binding. Binding to core RNAP induces or stabilizes an "open," active $\sigma$ conformation, optimal for promoter binding (Callaci et al., 1999; Schwartz et al., 2008; Vishwakarma et al., 2018). Canonically, RNAP activity at promoters is regulated through DNA-binding transcription factors (Browning and Busby, 2016) that recognize and bind to specific motifs on dsDNA (DB-TFs) and influence the initiation pathway steps after promoter binding (Figure 1A). A number of proteins, called $\sigma$-regulators in this review, have evolved to tune the structure of the $\sigma /$ core RNAP interaction, thus altering RNAP promoter selectivity and activity globally. These RNAPbinding transcription factors (RPB-TFs) bind to RNAP before the RNAP-promoter complex formation upon RNAP assembly. Consequently, RPB-TFs can influence all the ensuing steps of initiation and in some cases, also elongation and termination (Figures 1A,D). These proteins can be divided in two groups: (1) $\sigma$-activators (RbpA, Crl, GcrA, and GrgA) that target the $\sigma 2$ domain and consequently its interaction with the -10 element, and (2) б-repressors (Gp39, AsiA, P7, and Scc4) that target the $\sigma 4$ domain and consequently its interaction with -35 element (Table 1). All $\sigma$-repressor, but one, are phage-encoded proteins that appropriate the host transcriptional machinery during infection (Tabib-Salazar et al., 2019).

\section{ACTIVATORS TARGETING THE $\sigma 2$ DOMAIN}

\section{RbpA}

$\mathrm{RbpA}$ is a $\sim 14-\mathrm{kDa}$ protein specific to Actinomycetes sp. RbpA was discovered in Streptomyces coelicolor as a protein that is associated with the RNAP holoenzyme (Paget et al., 2001) and is required for rapid growth and confers basal levels of rifampicin resistance (Newell et al., 2006). Later studies in $M t b$ described RbpA as a $\sigma$-specific transcriptional activator implicated in the stress response (Hu et al., 2012, 2014) and essential for growth (Forti et al., 2011). RbpA binds to group 1 and group $2 \sigma$ subunits $\left(\sigma^{\mathrm{A}}\right.$ and $\sigma^{\mathrm{B}}$ in $M t b ; \sigma^{\mathrm{HrdB}}$ and $\sigma^{\mathrm{HrdA}}$ in $S$. coelicolor), but not to group 3 and group $4 \sigma$ subunits (Bortoluzzi et al., 2013; Tabib-Salazar et al., 2013; Hu et al., 2014). RbpA exerts multiple effects on transcription initiation. It stabilizes $\sigma$ interaction with core $M t b$ RNAP, promotes DNA melting, stabilizes RPo, and accelerates promoter escape ( $\mathrm{Hu}$ et al., 2012, 2014; Perumal et al., 2018). However, a recent study on the $\sigma^{\mathrm{A}}$-MtbRNAP holoenzyme suggests that RbpA inhibits promoter escape (Jensen et al., 2019). This discrepancy indicates that RbpA effect on transcription might be promoterspecific. RbpA structure comprises an unstructured N-terminal tail (NTT), a central RbpA core domain (RCD), and a C-terminal region called the $\sigma$-interacting domain (SID) (Figure $1 C$ ). RCD and SID are connected by a flexible loop called the basic linker (BL) (Bortoluzzi et al., 2013; Tabib-Salazar et al., 2013; Hubin et al., 2015). RbpA interacts with $\sigma 2$ via its SID, whereas BL (R79) interacts with promoter DNA upstream of the -10 element (Hubin et al., 2017). RbpA-SID interacts with three $\sigma 2$ regions: NCR, 1.2, and 2.3 (Figure 1C). RbpA tethers $\sigma^{\mathrm{A}}$ to core RNAP via the $\beta^{\prime}$-Zinc-binding domain (Hubin et al., 2015, 2017). Recent cryo-EM structures of $M t b$ RPo (Figure $2 \mathrm{C}$ ) showed that RbpANTT threads through the RNA exit channel into the active site cleft and interacts with the 3.2 region of $\sigma^{\mathrm{A}}$ and the DNA template strand at position -5 (Boyaci et al., 2019). However, RbpA-SID is sufficient for partial transcription activation (Hubin et al., 2015). The complex network of interactions between RbpA and key structural modules of RNAP explains why RbpA affects different steps of initiation, from RPo formation to promoter escape. Recent single-molecule Förster resonance energy transfer (smFRET) study showed that $M t b \sigma^{\mathrm{B}}$ adopts a closed, inactive conformation $(\sim 50 \AA$ distance between $\sigma 2$ and $\sigma 4$ ) even after assembly of the $\sigma^{\mathrm{B}}$-RNAP holoenzyme (Vishwakarma et al., 2018). During holoenzyme assembly, RbpA stabilizes (or induces) the open conformation of $\sigma^{\mathrm{B}}(\sim 83 \AA$ distance between $\sigma 2$ and $\sigma 4$ ), required for its tight binding to core $M t b$ RNAP and to promoter DNA (Figure 2A). Thus, RbpA acts as a chaperone to promote holoenzyme formation. This finding suggests that in the absence of RbpA, part of the $\sigma$-core RNAP interface cannot be formed, thus explaining 
A

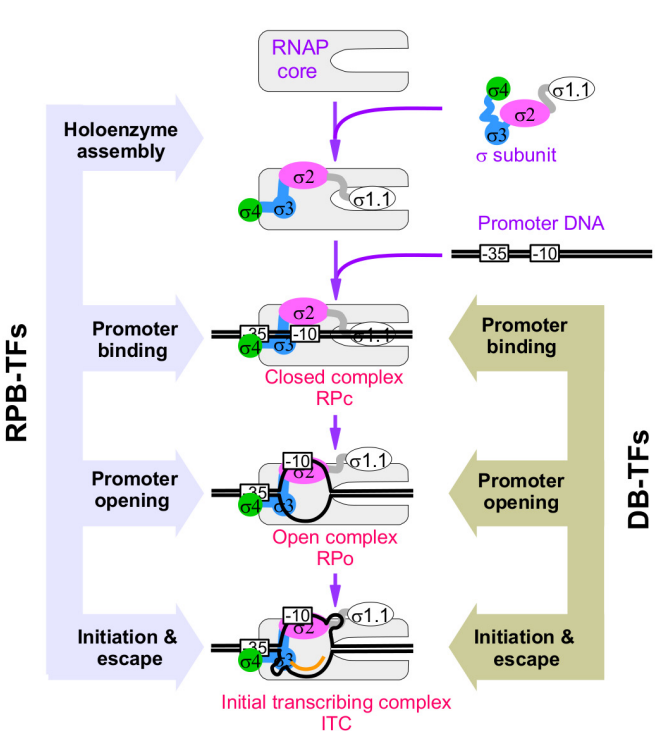

B

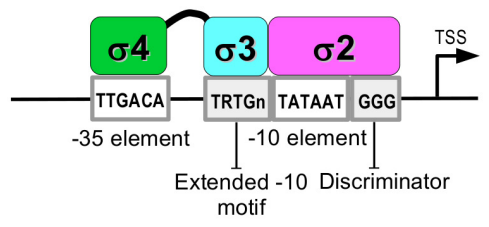

D

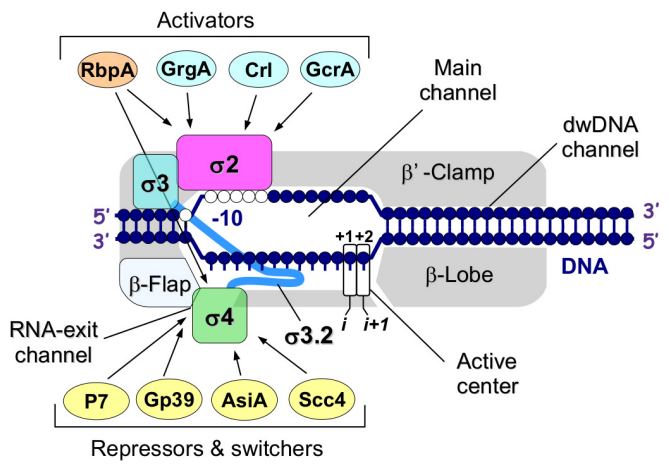

C

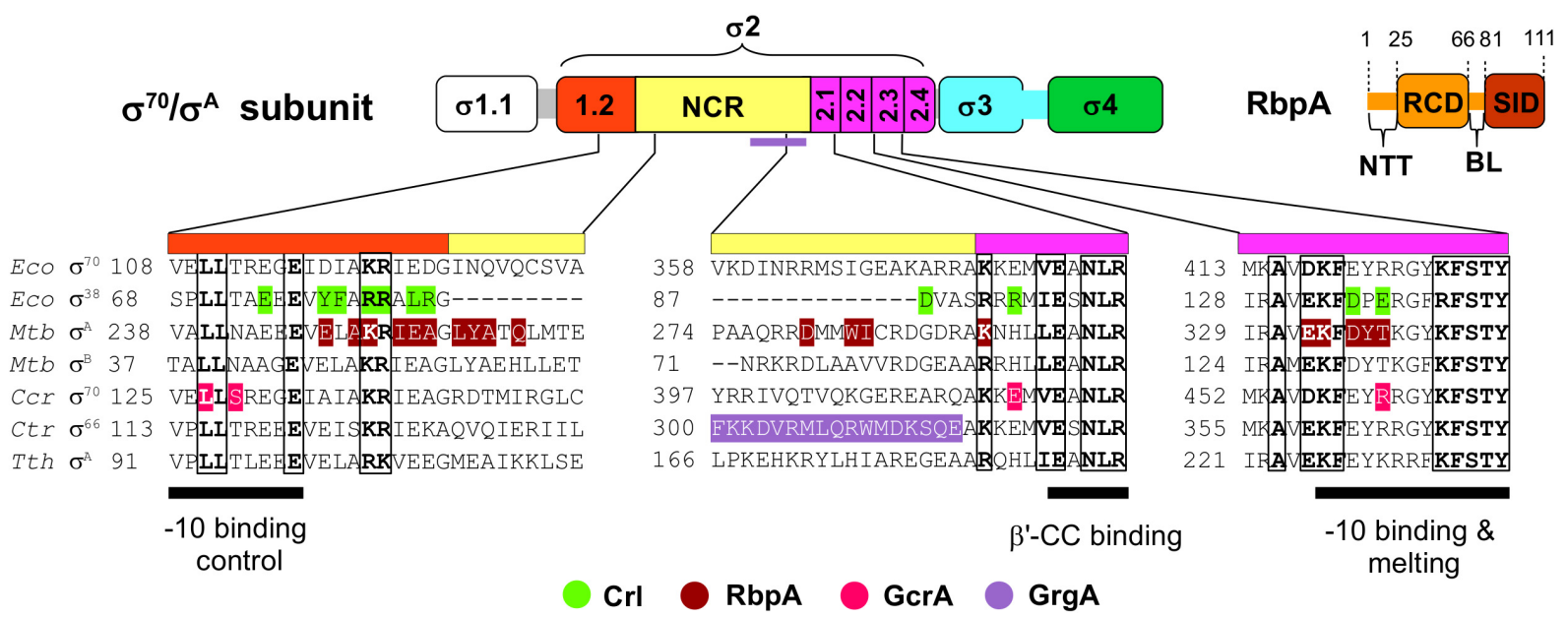

FIGURE 1 | The RNAP-binding $\sigma$-regulators and their interaction with RNAP. (A) Scheme of the main steps in transcription initiation. The steps regulated by RNAP-binding transcription factors (RPB-TFs) and DNA-binding transcription factors (DB-TFs) are indicated. (B) Basal promoter architecture (first described in E. coli) and interaction of its key elements with $\sigma$-domains. (C) Domain organization of the principal $\sigma$ subunits and RbpA. NCR - non-conserved region, NTT $\mathrm{N}$-terminal tail, RCD - RbpA core domain, BL - basic linker, SID - $\sigma$-interacting domain. Alignment of the $\sigma$ subunits from E. coli (Eco), M. tuberculosis (Mtb), C. crescentus (Ccr), C. trachomatis (Ctr), and T. thermophilus (Tth). Amino acid residues implicated in contacts with activators (bottom) are shown in color.

(D) Schematic presentation of the RNAP holoenzyme structure with the binding sites for the activators and repressors targeting domains $\sigma 2$ and $\sigma 4$, respectively.

the low stability of the $\sigma^{\mathrm{A}}$ and $\sigma^{\mathrm{B}} M t b \mathrm{RNAP}$ holoenzymes (Hu et al., 2012, 2014). On the basis of the high structural similarity between $\sigma^{\mathrm{A}}$ and $\sigma^{\mathrm{B}}$ we propose that the same activation mechanism works also for $\sigma^{\mathrm{A}}$. This conclusion is supported by the cryo-EM structure of Mycobacterium smegmatis $\sigma^{\mathrm{A}}$-RNAP holoenzyme lacking electron density for the domain $\sigma 4$. This indicates that $\sigma$ fluctuates between different conformational states (Kouba et al., 2019). The smFRET study on $\sigma^{\mathrm{B}}-M t b \mathrm{RNAP}$ also explains why $\mathrm{RbpA}$ is essential for transcription initiation at the $-10 /-35$ promoters and dispensable at the extended -10 promoters (Hu et al., 2012, 2014; Perumal et al., 2018). Indeed, RPo formation at the $-10 /-35$ promoters requires the distance between domains $\sigma 2$ and $\sigma 4$ to match the distance between the
-10 and -35 elements. This condition is dispensable for RPo formation at the extended -10 promoter. Therefore, regulation of the $\sigma$ conformational state by RbpA allows modulating RNAP promoter selectivity (Perumal et al., 2018).

\section{Crl}

Crl is a $\sim 16-\mathrm{kDa}$ protein from $\gamma$-proteobacteria, initially identified in $E$. coli as an activator of genes implicated in curli fimbriae production (Arnqvist et al., 1992). Crl binds to stationary phase $\sigma^{S}$ and activates $\sigma^{S}$-RNAP-mediated transcription, independently of the promoter sequence (Pratt and Silhavy, 1998; Bougdour et al., 2004). Although Crl does not bind to $\sigma^{70}$ because of the steric clash with 
TABLE 1 | Properties of the RNAP-binding $\sigma$-regulators.

\begin{tabular}{|c|c|c|c|c|c|c|c|}
\hline Name & Phylum/organism & Targeted $\sigma$ & Binding site & DNA interaction & Regulated process & Mode of action & Structures/PDB code \\
\hline \multicolumn{8}{|l|}{$\sigma$-activators } \\
\hline RbpA & $\begin{array}{l}\text { Actinobacteria/Mycobacterium } \\
\text { tuberculosis }\end{array}$ & $\sigma^{\mathrm{A}}, \sigma^{\mathrm{B}}$ & $\begin{array}{l}\sigma 2, \beta^{\prime} \text {-clamp/RNA-exit } \\
\text { channel }\end{array}$ & Nonspecific & $\begin{array}{l}\text { - Growth } \\
\text { - Stress response } \\
\text { - Stationary phase }\end{array}$ & $\begin{array}{l}\sigma \text {-RNAP assembly } \\
\text { (chaperon); Stimulates } \\
\text { RPo formation; } \\
\text { Stimulates promoter } \\
\text { escape }\end{array}$ & $\begin{array}{l}\text { RbpA- } \sigma^{A}-R P o / 6 C 04,5 T W 1 \\
\text { 5VI5; RbpA- } \sigma^{A}-R N A P / 6 C 05\end{array}$ \\
\hline $\mathrm{Crl}$ & $\begin{array}{l}\gamma \text {-Proteobacteria/Escherichia } \\
\text { coli }\end{array}$ & $\sigma^{S}$ & $\sigma 2 / \beta^{\prime}-\mathrm{CT}$ & No & $\begin{array}{l}\text { - Stress response } \\
\text { - Stationary phase }\end{array}$ & $\begin{array}{l}\sigma \text {-RNAP assembly } \\
\text { (chaperon); Stimulates } \\
\text { RPo formation }\end{array}$ & Crl-ITC5/6KJ6; Crl-RPo/6OMF \\
\hline GcrA & $\begin{array}{l}\alpha \text {-Proteobacteri/Caulobacter } \\
\text { crescentus }\end{array}$ & $\sigma^{\mathrm{A}}$ & $\sigma 2$ & $\begin{array}{l}\text { Methylated DNA } \\
\left(\mathrm{m}^{6} \mathrm{~A}\right)\end{array}$ & - Cell cycle & $\begin{array}{l}\text { Stimulates RPo } \\
\text { formation at methylated } \\
\text { promoters }\end{array}$ & GcrA- $\sigma^{\mathrm{A}} / 5 \mathrm{YIX}$ \\
\hline GrgA & $\begin{array}{l}\text { Chlamydiae/Chlamydia } \\
\text { trachomatis }\end{array}$ & $\sigma^{A}, \sigma^{28}$ & $\sigma 2$ & Nonspecific & Unknown & $\begin{array}{l}\text { Activates transcription } \\
\text { initiation }\end{array}$ & - \\
\hline \multicolumn{8}{|l|}{$\sigma$-repressors } \\
\hline Scc4 (CT663) & $\begin{array}{l}\text { Chlamydiae/Chlamydia } \\
\text { trachomatis }\end{array}$ & $\sigma^{\mathrm{A}}$ & $\sigma 4 / \beta-F L A P$ & No & $\begin{array}{l}\text { - Growth } \\
\text { - Infection }\end{array}$ & $\begin{array}{l}\text { Inhibits transcription } \\
\text { initiation at }-10 /-35 \\
\text { promoters (likely by } \sigma 4 \\
\text { displacement) }\end{array}$ & - \\
\hline Gp39 & $\begin{array}{l}\text { Deinococcus- } \\
\text { Thermus/Thermus } \\
\text { thermophilus Phage P23-45 }\end{array}$ & $\sigma^{A}$ & $\sigma 4 / \beta-F L A P$ & No & - Phage transcription & $\begin{array}{l}\text { Inhibits RPo formation } \\
\text { by } \sigma 4 \text { displacement; } \\
\text { Stimulates elongation. } \\
\text { Anti-terminator function }\end{array}$ & RNAP-Gp39/3WOD \\
\hline AsiA & $\begin{array}{l}\gamma \text {-Proteobacteria/Escherichia } \\
\text { coli Phage T4 }\end{array}$ & $\sigma^{70}$ & $\sigma 4 / \beta-F L A P$ & Nonspecific & - Phage transcription & $\begin{array}{l}\text { Inhibits host RPo } \\
\text { formation by } \sigma 4 \\
\text { appropriation ( } \sigma 4 \\
\text { displacement); } \\
\text { Stimulates phage RPo } \\
\text { formation }\end{array}$ & RPo-AsiA-MotA/6K4Y \\
\hline P7 & $\begin{array}{l}\gamma \text {-Proteobacteria/Xanthomonas } \\
\text { oryzae Phage Xp10 }\end{array}$ & No & $\begin{array}{l}\beta^{\prime}-\mathrm{NTD} / \beta-\mathrm{FLAP} / \mathrm{RNA}- \\
\text { exit } \\
\text { channel }\end{array}$ & Unknown & - Phage transcription & $\begin{array}{l}\text { Inhibits RPo formation } \\
\text { by } \sigma 4 \text { displacement; } \\
\text { Stimulates elongation; } \\
\text { Anti-terminator function }\end{array}$ & P7-TEC/6J9F \\
\hline
\end{tabular}


A

RbpA-driven activation in Mycobacteria sp.

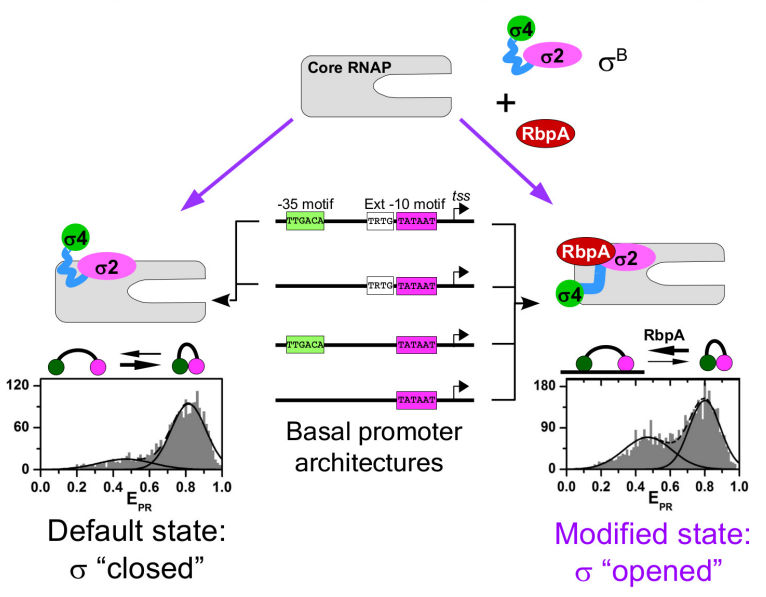

C

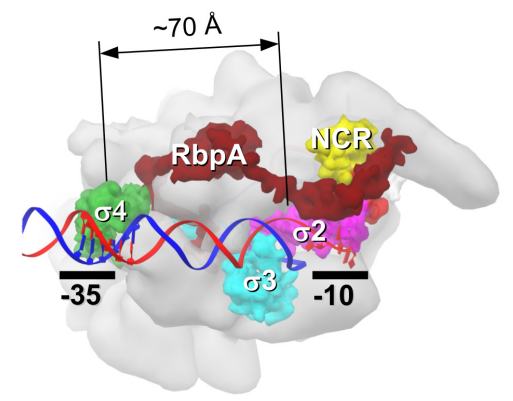

M. tuberculosis $\sigma^{\mathrm{A}}$-RNAP-RbpA $\sigma$ "opened"
B

Gp39-driven repression in Thermus sp.

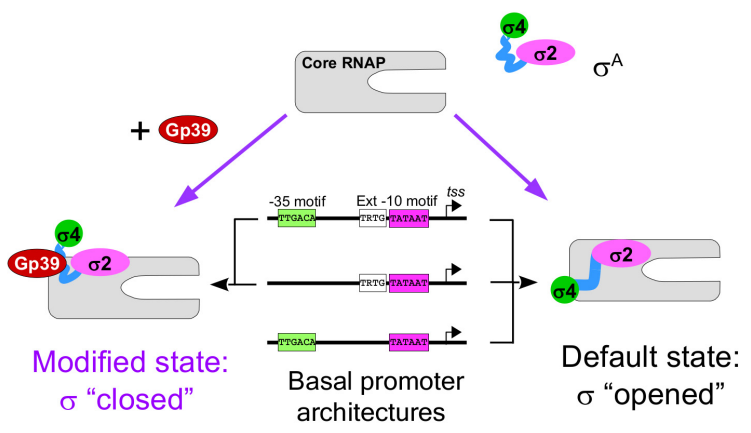

D

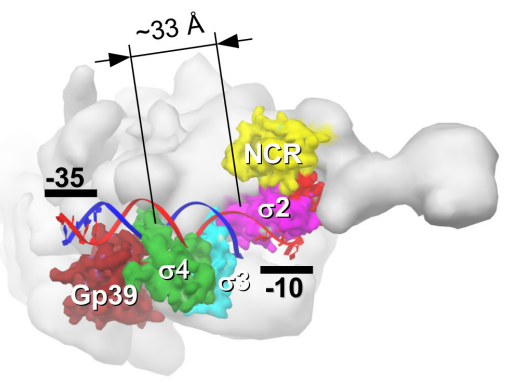

T. thermophilus $\sigma^{\mathrm{A}}$-RNAP-Gp39 $\sigma$ "closed"

FIGURE 2 | Regulation of the $\sigma$ subunit conformational states by RbpA and Gp39. (A) Model representing the mechanism of RbpA-driven transcription activation in Mycobacteria sp. At the bottom, the histograms show the smFRET efficiencies (EPR) distributions for the double-labeled $\sigma^{B}$ subunit in the RNAP holoenzyme without (left) and with RbpA (right) (data from Vishwakarma et al., 2018). (B) Model representing the mechanism of gp39-driven transcription repression in T. thermophilus. (C) Structure of the MtbRNAP- $\sigma^{A}$ RPo in complex with RbpA [Protein Data Bank (PDB) code: 6EDT]. (D) Structure of the Tht RNAP- $\sigma^{A}$ RPo in complex with gp39 [Protein Data Bank (PDB) code: 3WOD]. The dimension lines show distances between C $\alpha$ atoms of homologous residues in domain $\sigma 2$ (Mtb T356, Tht N248) and domain $\sigma 4$ (Mtb G497, Tht G391).

$\sigma^{70}$-NCR (Cartagena et al., 2019) it can activate $\sigma^{70}$-dependent transcription (Gaal et al., 2006). As observed for RbpA, Crl facilitates transcription initiation by stabilizing the $\sigma^{\mathrm{S}}$-RNAP holoenzyme and stimulating RPo formation (Banta et al., 2013; Xu et al., 2019). Two high resolution cryo-EM-based structures of Crl- $\sigma^{\mathrm{S}}$-RNAP RPo have been recently described (Cartagena et al., 2019; Xu et al., 2019). Cartagena et al., suggested that Crl stabilizes $\sigma^{\mathrm{S}}$-RNAP by tethering $\sigma^{\mathrm{S}}$ directly to RNAP though contacts with the $\beta^{\prime}$-clamp-toe domain ( $\beta^{\prime}$-CT, residues 144-179). Based on structure and hydrogen-deuterium exchange mass spectrometry analysis of $\sigma^{S}$ conformation, $\mathrm{Xu}$ et al. (2019) suggested that $\mathrm{Crl}$ acts as a chaperone that facilitates the $\sigma^{\mathrm{S}}$-RNAP holoenzyme assembly mainly by modifying $\sigma 2$ conformation, but not through its contacts with the $\beta^{\prime}$-clamp. In addition, interaction of $\mathrm{Crl}$ residue R51 with residues D135 and E137 in the "specificity loop" of region 2.3 of $\sigma$ (Figure 1C) stabilizes its optimal conformation for binding to the -10 element ssDNA. This interaction promotes RPo formation. It is not known whether Crl plays any role in promoter escape. However, the finding that the $\beta^{\prime}$ $\mathrm{CT} / \sigma^{70}$-NCR interaction antagonizes the $\sigma 2 / \beta^{\prime}$-clamp interaction and facilitaes promoter escape (Leibman and Hochschild, 2007) suggests this possibility (Banta et al., 2013; Cartagena et al., 2019).

\section{GcrA}

GcrA (173-aa) is a transcription factor from Caulobacter crescentus that is well conserved in $\alpha$-proteobacteria. GcrA forms a stable complex with $\sigma^{\mathrm{A}}$-RNAP, recruits RNAP to methylated $\left(\mathrm{m}^{6} \mathrm{~A}\right)$ promoters, and activates the expression of $\sim 200$ genes that play an important role in cell cycle regulation during swarmer-tostalked cell transition (Holtzendorff et al., 2004; Haakonsen et al., 2015). Analysis of the promoter binding kinetics demonstrated that GcrA increases RNAP affinity for the promoter and the rate of RPc isomerization to RPo (Haakonsen et al., 2015). GcrA is composed of two domains: the N-terminal DNA-binding domain (GcrA-DBD, residues 1-45) that recognizes methylated promoter DNA, and the C-terminal $\sigma$-interacting domain (GcrA-SID, residues 108-173) that binds to $\sigma 2$ (Figure 1C). GcrA-DBD and GcrA-SID are connected by an unstructured linker (residues 46107). Recent crystal structures of the GcrA-SID- $\sigma^{A}$ complex and the GcrA-DBD-DNA complex revealed details of its interactions 
with RNAP and the promoter (Wu et al., 2018). Structural studies on the full length protein and its complex with RNAP are now needed to decipher GcrA mechanism of action.

\section{GrgA}

GrgA (ORF CTL0766, 288-aa) is a transcription factor from the human pathogen Chlamydia trachomatis. GrgA activates $\sigma^{\mathrm{A}}$ - (also known as $\sigma^{66}$ ) and $\sigma^{28}$-dependent transcription by interacting with $\sigma-\mathrm{NCR}$ and binding to DNA in a non-sequencespecific manner (Bao et al., 2012; Desai et al., 2018). The GrgA binding site on $\sigma^{\mathrm{A}}$ was mapped to residues 269-316 (Bao et al., 2012) (Figure 1C). The detailed mechanism of GrgA action and its role in gene regulation remain obscure. GrgA is specific to Chlamydia species, and has not been found in any other organism. Therefore, it might be a good target for developing highly selective anti-chlamydial drugs (Zhang et al., 2019).

\section{REPRESSORS TARGETING THE $\sigma 4$ DOMAIN}

\section{Gp39 of Phage P23-45}

Gp39, a $\sim 16-\mathrm{kDa}$ protein encoded by the Thermus thermophilus phage P23-45, binds to the host $\sigma^{\mathrm{A}}$-RNAP holoenzyme and inhibits transcription from $-10 /-35$ class promoters. Transcription of the middle and late promoters of P23-45, which belong to the extended -10 class, is less affected (Berdygulova et al., 2011; Tagami et al., 2014). Gp39 blocks transcription initiation probably at the step of RPc formation that depends on the $\sigma 4 /-35$ element contact. Besides its effect on initiation, gp39 also displays anti-termination activity (Berdygulova et al., 2012) suggesting that the $\sigma$ subunit is not essential for its binding to RNAP. The crystal structure of the $\sigma^{\mathrm{A}}$-RNAP-gp39 complex (Tagami et al., 2014) reveled that gp39 binds to the RNAP $\beta$ flap and to the $\sigma 4$ domain and induces a $\sim 45 \AA$ displacement of the $\sigma 4$ relative to its default position in the RNAP holoenzyme (Figures 2B,D). This conformational change in the $\sigma^{\mathrm{A}}$ subunit explains the selectivity of the RNAP-gp39 complex toward the extended -10 promoters.

\section{AsiA of Phage T4}

AsiA is 90 -aa protein of the E. coli phage T4. AsiA employs a mechanism called $\sigma$ appropriation to reprogram the host RNAP. AsiA forms a stable complex with $\sigma^{70}$ before holoenzyme assembly (Hinton et al., 1996; Hinton and Vuthoori, 2000) and thus inhibits transcription from the $-10 /-35$ class promoters. Conversely, transcription from the extended -10 promoters is less affected (Severinovaa et al., 1998). At the same time, AsiA acts as a co-activator of the phage activator protein MotA, required for binding to the T4 middle promoter. The $\sigma$ appropriation complex, which includes $\sigma^{70}$, RNAP, AsiA and MotA, recognizes the MotA-box that replaces the -35 element at the T4 middle promoters. NMR solution structures of the AsiA- $\sigma 4$ complex demonstrated that AsiA remodels $\sigma 4$ making impossible its binding to the -35 element and its interaction with $\beta$-flap (Simeonov et al., 2003; Lambert et al., 2004). A recent cryoEM structure of the $\sigma^{70}$-RNAP-AsiA-MotA RPo revealed the detailed mechanism of $\sigma$ appropriation (Shi et al., 2019). AsiA binds to and remodels the structure of the $\sigma$ region 3.2 and $\sigma 4$, displaces $\sigma 4$, and takes its place. This allows MotA recruitment and RPo formation. In addition, AsiA interaction with upstream dsDNA stabilizes RPo.

\section{P7 of Phage Xp10}

P7 is a small, $\sim 8-\mathrm{kDa}$, globular protein encoded by the lytic bacteriophage Xp10 that infects the Gram-negative bacterium Xanthomonas oryzae, which causes rice blight. At a later stage of infection, P7 shuts off the host gene transcription in favor of phage gene transcription by the Xp10 singlesubunit RNAP (Nechaev et al., 2002; Liu et al., 2014). P7 forms a stable complex with the host $\sigma^{70}$-RNAP holoenzyme and inhibits RPo formation at $-10 /-35$ promoters and to a lesser extent, at the extended -10 promoters. Luminescence resonance energy transfer (LRET) measurements demonstrated that in the P7- $\sigma^{70}$-RNAP complex, the $\sigma^{70}$ subunit adopts a closed or partially closed conformation (Nechaev et al., 2002). The finding that P7 also binds to RNAP harboring the structurally distinct $\sigma^{54}$ (Brown et al., 2016) suggests that the $\sigma$ subunit is not essential for its interaction with RNAP. Indeed, P7 can also modulate post-initiation steps of transcription, such as pausing and intrinsic termination (Nechaev et al., 2002; Zenkin et al., 2015 You et al., 2019). A recently solved cryoEM structure of P7 in the elongation complex (You et al., 2019) reveled that P7 binds to the RNA-exit channel at the place of $\sigma 4$, and thus makes impossible the formation of the "open" $\sigma$ conformation essential for RPo formation at $-10 /-35$ class promoters. The lack of $\sigma 4$-RNAP contact should decrease the overall stability of the holoenzyme, thus explaining the dissociation of $\sigma$ from the P7-RNAP complex observed in biochemical experiments (Liu et al., 2014). It has been proposed that P7 induces the closed conformation of the RNAP clamp, and thus inhibits RPo formation (You et al., 2019). However, it is unlikely that such mechanism takes place at $-10 /-35$ promoters. Indeed, according to the P7RNAP complex structure, P7 should inhibit the interaction of $\sigma 4$ with the -35 element, which is required for initial RNAP binding to the promoter (RPc formation). Clamp closing starts to play a role during $\mathrm{RPc}$ isomerization to $\mathrm{RPo}$, the step following recognition of the -35 element. Thus, it is more likely that P7-mediated $\sigma^{70}$ remodeling inhibits the $\sigma 4 /-35$ element interaction and consequently RPc formation, as observed for the $\sigma^{54}$-RNAP holoenzyme (Brown et al., 2016). However, P7induced clamp closing might play a role when the -35 element recognition is bypassed.

\section{Scc4 (CT663) From Chlamydia trachomatis}

Scc4 (ORF CT663) is a $\sim 15-\mathrm{kDa}$ protein from the human pathogen C. trachomatis. Scc4 forms a heterodimer with Scc1, and both are type III secretion chaperons implicated in the regulation of cell growth and intracellular infection (Hanson et al., 2015). Scc4 was identified in a two-hybrid screen for regulators that interact with $C$. trachomatis RNAP $\beta$-FLAP 
(Rao et al., 2009). Scc4 binds to RNAP $\beta$-FLAP tip helix and also interacts with the $\sigma 4$ domain of the principal $\sigma^{\mathrm{A}}$ subunit. It can also interact with the $\sigma 4$ domain of E. coli $\sigma^{70}$ that exhibits $60 \%$ amino acid identity with the $\sigma 4$ of $\sigma^{\mathrm{A}}$, but does not interact with the $\sigma 4$ of C. trachomatis $\sigma^{28}$ (Group 3). Scc4 inhibits transcription initiation from $-10 /-35$ class promoters, but not from extended -10 type promoters. Although structural studies are needed to determine the mechanism of inhibition, on the basis of similarities with the mechanism of action of the phage proteins we hypothesize that Scc4 disrupts the $\sigma 4 /$ Flap interaction and prevents RPo formation at $-10 /-35$ promoters.

\section{CONCLUSION}

We can draw two basic principles of transcription regulation by RPB-TFs: positive regulation through strengthening of $\sigma 2 / \beta^{\prime}$-clamp/-10 element interactions, and negative regulation through weakening of $\sigma 4 / \beta$-flap/-35 element interactions. All contacts made by the three $\sigma$-activators RbpA, Crl and GcrA overlap and are clustered in four $\sigma$ regions $(\sigma 1.2, \sigma-\mathrm{NCR}, \sigma 2.1$ and $\sigma 2.3$ ) that are responsible for core RNAP binding and -10 element recognition/melting (Figure 1C). Consequently, all these activators act through a similar mechanism. They strengthen $\sigma /$ RNAP interaction and stimulate RPo formation, the rate limiting step in transcription initiation. The only exception is GrgA the binding site of which was mapped entirely to $\sigma$ NCR and thus may have a different mechanism of action. However, in the absence of a detailed biochemical and structural characterization, it cannot be excluded that GrgA contacts other regions besides $\sigma$-NCR.

At least for RbpA, the stimulation of the "closed-to-open" transition is part of the $\sigma$ activation mechanism required for

\section{REFERENCES}

Arnqvist, A., Olsén, A., Pfeifer, J., Russell, D. G., and Normark, S. (1992). The Crl protein activates cryptic genes for curli formation and fibronectin binding in Escherichia coli HB101. Mol. Microbiol. 6, 2443-2452. doi: 10.1111/j.1365-2958. 1992.tb01420.x

Banta, A. B., Chumanov, R. S., Yuan, A. H., Lin, H., Campbell, E. A., Burgess, R. R., et al. (2013). Key features of $\sigma \mathrm{S}$ required for specific recognition by Crl, a transcription factor promoting assembly of RNA polymerase holoenzyme. Proc. Natl. Acad. Sci. U.S.A. 110, 15955-16960. doi: 10.1073/pnas.1311642110

Bao, X., Nickels, B. E., and Fan, H. (2012). Chlamydia trachomatis protein GrgA activates transcription by contacting the nonconserved region of $\sigma 66$. Proc. Natl. Acad. Sci. U.S.A. 109, 16870-16875. doi: 10.1073/pnas.12073 00109

Barne, K. A., Bown, J. A., Busby, S. J., and Minchin, S. D. (1997). Region 2.5 of the Escherichia coli RNA polymerase $\sigma 70$ subunit is responsible for the recognition of the 'extended-10' motif at promoters. EMBO J. 16, 4034-4040. doi: 10.1093/emboj/16.13.4034

Berdygulova, Z., Esyunina, D., Miropolskaya, N., Mukhamedyarov, D., Kuznedelov, K., Nickels, B. E., et al. (2012). A novel phage-encoded transcription antiterminator acts by suppressing bacterial RNA polymerase pausing. Nucleic Acids Res. 40, 4052-4063. doi: 10.1093/nar/gkr1285

Berdygulova, Z., Westblade, L. F., Florens, L., Koonin, E. V., Chait, B. T., and Ramanculov, E. (2011). Temporal regulation of gene expression of the Thermus thermophilus bacteriophage P23-45. J. Mol. Biol. 405, 125-142. doi: 10.1016/j. jmb.2010.10.049 efficient transcription initiation at the $-10 /-35$ class promoters, but not at the extended -10 class promoters (Vishwakarma et al., 2018). It remains to be explored whether Crl, GcrA and GrgA can affect the relative movement of the $\sigma 2$ and $\sigma 4$ domains. Remarkably, all $\sigma$-repressors mentioned here act as antagonists to RbpA-type activation by destabilizing the $\sigma 4 / \beta$-flap interaction, and should favor the "open-to-closed" transition in the $\sigma$ subunit. Consequently, $\sigma$-repressor-modified RNAP cannot initiate transcription at the $-10 /-35$ class promoters, but only at the extended -10 class promoters (Figure $2 \mathbf{A}, \mathbf{B}$ ).

The $\sigma$-activators and $\sigma$-repressors illustrate how $\sigma$ conformational dynamics, controlled by contacts with core RNAP, can be used for fine-tuning transcription in a lineagespecific manner. Considering the huge diversity in lifestyles of bacterial species, the number of the currently known $\sigma$-regulators of bacterial origin is strikingly low. The reason might be that most of these proteins are of small size and are not easy to detect. Yet, their discovery in pathogenic bacteria may offer new targets for developing pathogen-specific drugs. We expect that the number of the described $\sigma$-regulators and the diversity of regulatory mechanisms will continue to grow.

\section{AUTHOR CONTRIBUTIONS}

$\mathrm{KB}$ and RV wrote the manuscript. Both authors contributed to the article and approved the submitted version.

\section{FUNDING}

This work was supported by the grant from the French National Research Agency [MycoMaster ANR-16-CE11-0025-01].

Bortoluzzi, A., Muskett, F. W., Ross, W., Gourse, R. L., Campbell, E. A., Waters, L. C., et al. (2013). Mycobacterium tuberculosis RNA polymerase-binding protein A (RbpA) and its interactions with sigma factors. J. Biol. Chem. 288, 14438-14450. doi: 10.1074/jbc.M113.459883

Bougdour, A., Lelong, C., and Geiselmann, J. (2004). Crl, a low temperatureinduced protein in Escherichia coli that binds directly to the stationary phase sigma subunit of RNA polymerase. J. Biol. Chem. 279, 19540-19550. doi: 10. 1074/jbc.M314145200

Boyaci, H., Chen, J., Jansen, R., Darst, S. A., and Campbell, E. A. (2019). Structures of an RNA polymerase promoter melting intermediate elucidate DNA unwinding. Nature 565, 382-385. doi: 10.1038/s41586-018-0840-5

Brown, D. R., Sheppard, C. M., Burchell, L., Matthews, S., and Wigneshweraraj, S. (2016). The Xp10 bacteriophage protein P7 inhibits transcription by the major and major variant forms of the host RNA polymerase via a common mechanism. J. Mol. Biol. 428, 3911-3919. doi: 10.1016/j.jmb.2016.08.004

Browning, D. F., and Busby, S. J. W. (2016). Local and global regulation of transcription initiation in bacteria. Nat. Rev. Microbiol. 14, 638-650. doi: 10. 1038/nrmicro.2016.103

Buc, H., and McClure, W. R. (1985). Kinetics of open complex formation between Escherichia coli RNA polymerase and the lac UV5 promoter. Evidence for a sequential mechanism involving three steps. Biochemistry 24, 2712-2723. doi: 10.1021/bi00332a018

Burr, T., Mitchell, J., Kolb, A., Minchin, S., and Busbya, S. (2000). DNA sequence elements located immediately upstream of the -10 hexamer in Escherichia coli promoters: a systematic study. Nucleic Acids Res. 28, 1864-1870. doi: 10.1093/ nar/28.9.1864 
Callaci, S., Heyduk, E., and Heyduk, T. (1999). Core RNA polymerase from E. coli induces a major change in the domain arrangement of the $\sigma 70$ subunit. Mol. Cell 3, 229-238. doi: 10.1016/s1097-2765(00)80313-5

Cartagena, A. J., Banta, A. B., Sathyan, N., Ross, W., Gourse, R. L., Campbell, E. A., et al. (2019). Structural basis for transcription activation by Crl through tethering of $\sigma$ S and RNA polymerase. Proc. Natl. Acad. Sci. U.S.A. 116, 1892318927. doi: 10.1073/pnas.1910827116

Cortes, T., Schubert, O. T., Rose, G., Arnvig, K. B., Comas, I., Aebersold, R., et al. (2014). Genome-wide mapping of transcriptional start sites defines an extensive leaderless transcriptome in Mycobacterium tuberculosis. Cell Rep. 5, 1121-1131. doi: 10.1016/j.celrep.2013.10.031

Desai, M., Wurihan, W., Di, R., Fondell, J. D., Nickels, B. E., Bao, X., et al. (2018). A role for GrgA in regulation of $\sigma 28$ dependent transcription in the obligate intracellular bacterial pathogen Chlamydia trachomatis. J. Bacteriol. 200:e00298-18.

Feklistov, A., and Darst, S. A. (2011). Structural basis for promoter -10 element recognition by the bacterial RNA polymerase $\sigma$ subunit. Cell 147, 1257-1269. doi: 10.1016/j.cell.2011.10.041

Feklistov, A., Sharon, B. D., Darst, S. A., and Gross, C. A. (2014). Bacterial sigma factors: a historical, structural, and genomic perspective. Annu. Rev. Microbiol. 68, 357-376. doi: 10.1146/annurev-micro-092412-155737

Forti, F., Mauri, V., Deho, G., and Ghisotti, D. (2011). Isolation of conditional expression mutants in Mycobacterium tuberculosis by transposon mutagenesis. Tuberculosis 91, 569-578. doi: 10.1016/j.tube.2011.07.004

Gaal, T., Mandel, M. J., Silhavy, T. J., and Gourse, R. L. (2006). Crl facilitates RNA polymerase holoenzyme formation. J. Bacteriol. 188, 7966-7970. doi: 10.1128/jb.01266-06

Gruber, T. M., and Gross, C. A. (2003). Multiple sigma subunits and the partitioning of bacterial transcription space. Annu. Rev. Microbiol. 57, 441-466. doi: 10.1146/annurev.micro.57.030502.090913

Haakonsen, D. L., Yuan, A. H., and Laub, M. T. (2015). The bacterial cell cycle regulator GcrA is a $\sigma 70$ cofactor that drives gene expression from a subset of methylated promoters. Genes Dev. 29, 2272-2286. doi: 10.1101/gad.270660.115

Hanson, B. R., Slepenkin, A., Peterson, E. M., and Tan, M. (2015). Chlamydia trachomatis type III secretion proteins regulate transcription. J. Bacteriol. 197, 3238-3244. doi: 10.1128/jb.00379-15

Hinton, D. M., Amegadzie, R. M., Gerber, J. S., and Sharma, M. (1996). Bacteriophage T4 middle transcription system: T4-modified RNA polymerase; AsiA, a $\sigma 70$ binding protein; and transcriptional activator MotA. Methods Enzymol. 274, 43-57. doi: 10.1016/s0076-6879(96)74007-7

Hinton, D. M., and Vuthoori, S. (2000). Efficient inhibition of Escherichia coli RNA polymerase by the bacteriophage T4 AsiA protein requires that AsiA binds first to free $\sigma 70$. J. Mol. Biol. 304, 731-739. doi: 10.1006/jmbi.2000.4113

Holtzendorff, J., Hung, D., Brende, P., Reisenauer, A., Viollier, P. H., McAdams, H. H., et al. (2004). Oscillating global regulators control the genetic circuit driving a bacterial cell cycle. Science 304, 983-987. doi: 10.1126/science. 1095191

Hu, Y., Morichaud, Z., Chen, S., Leonetti, J. P., and Brodolin, K. (2012). Mycobacterium tuberculosis RbpA protein is a new type of transcriptional activator that stabilizes the $\sigma \mathrm{A}$-containing RNA polymerase holoenzyme. Nucleic Acids Res. 40, 6547-6557. doi: 10.1093/nar/gks346

Hu, Y., Morichaud, Z., Perumal, A. S., Roquet-Baneres, F., and Brodolin, K. (2014). Mycobacterium RbpA cooperates with the stress-response $\sigma \mathrm{B}$ subunit of RNA polymerase in promoter DNA unwinding. Nucleic Acids Res. 42, 10399-10408. doi: 10.1093/nar/gku742

Hubin, E. A., Fay, A., Xu, C., Bean, J. M., Saecker, R. M., Glickman, M. S., et al. (2017). Structure and function of the mycobacterial transcription initiation complex with the essential regulator RbpA. eLife 6:e22520. doi: 10.7554/eLife. 22520

Hubin, E. A., Tabib-Salazar, A., Humphrey, L. J., Flack, J. E., Olinares, P. D. B., Darst, S. A., et al. (2015). Structural, functional, and genetic analyses of the actinobacterial transcription factor RbpA. Proc. Natl. Acad. Sci. U.S.A. 112, 7171-7176. doi: 10.1073/pnas.1504942112

Jensen, D., Manzano, A. R., Rammohan, J., Stallings, C. L., and Galburt, E. A. (2019). CarD and RbpA modify the kinetics of initial transcription and slow promoter escape of the Mycobacterium tuberculosis RNA polymerase. Nucleic Acids Res. 13, 6685-6698. doi: 10.1093/nar/gkz449
Keilty, S., and Rosenberg, M. (1987). Constitutive function of a positively regulated promoter reveals new sequences essential for activity. J. Biol. Chem. 262, 6389-6395.

Kouba, T., Pospíšil, J., Hnilicová, J., Šanderová, H., Barvík, I., and Krásn $\iota$, L. (2019). The core and holoenzyme forms of RNA polymerase from Mycobacterium smegmatis. J. Bacteriol. 201:e00583-18.

Kumar, A., Malloch, R. A., Fujita, N., Smillie, D. A., Ishihama, A., and Hayward, R. S. (1993). The minus 35-recognition region of Escherichia coli sigma 70 is inessential for initiation of transcription at an "extended minus 10 " promoter. J. Mol. Biol. 232, 406-418. doi: 10.1006/jmbi.1993.1400

Lambert, L. J., Wei, Y., Schirf, V., Demeler, B., and Werner, M. H. (2004). T4 AsiA blocks DNA recognition by remodeling $\sigma 70$ region 4. EMBO J. 23, 2952-2962. doi: 10.1038/sj.emboj.7600312

Leibman, M., and Hochschild, A. (2007). A $\sigma$-core interaction of the RNA polymerase holoenzyme that enhances promoter escape. EMBO J. 26, 15791590. doi: 10.1038/sj.emboj.7601612

Li, L., Molodtsov, V., Lin, W., Ebright, R. H., and Zhang, Y. (2020). RNA extension drives a stepwise displacement of an initiation-factor structural module in initial transcription. Proc. Natl. Acad. Sci. U.S.A. 117, 5801-5809. doi: 10.1073/ pnas. 1920747117

Liu, B., Shadrin, A., Sheppard, C., Mekler, V., Xu, Y., Severinov, K., et al. (2014). A bacteriophage transcription regulator inhibits bacterial transcription initiation by $\sigma$-factor displacement. Nucleic Acids Res. 42, 4294-4305. doi: 10.1093/nar/ gku080

Mitchell, J. E., Zheng, D., Busby, S. J. W., and Minchin, S. D. (2003). Identification and analysis of 'extended -10' promoters in Escherichia coli. Nucleic Acids Res. 31, 4689-4695. doi: 10.1093/nar/gkg694

Morichaud, Z., Chaloin, L., and Brodolin, K. (2016). Regions 1.2 and 3.2 of the RNA polymerase $\sigma$ subunit promote DNA melting and attenuate action of the antibiotic lipiarmycin. J. Mol. Biol. 428, 463-476. doi: 10.1016/j.jmb.2015.12. 017

Narayanan, A., Vago, F. S., Li, K., Qayyum, M. Z., Yernool, D., Jiang, W., et al. (2018). Cryo-EM structure of Escherichia coli $\sigma 70$ RNA polymerase and promoter DNA complex revealed a role of $\sigma$ non-conserved region during the open complex formation. J. Biol. Chem. 293, 7367-7375. doi: 10.1074/jbc. RA118.002161

Nechaev, S., Yuzenkova, Y., Niedziela-Majka, A., Heyduk, T., and Severinov, K. (2002). A novel bacteriophage-encoded RNA polymerase binding protein inhibits transcription initiation and abolishes transcription termination by host RNA polymerase. J. Mol. Biol. 320, 11-22. doi: 10.1016/s0022-2836(02)00420-5

Newell, K. V., Thomas, D. P., Brekasis, D., et al. (2006). The RNA polymerasebinding protein RbpA confers basal levels of rifampicin resistance on Streptomyces coelicolor. Mol. Microbiol. 60, 687-696. doi: 10.1111/j.1365-2958. 2006.05116.x

Österberg, S., del Peso-Santos, T., and Shingler, V. (2011). Regulation of alternative sigma factor use. Annu. Rev. Microbiol. 65, 37-55. doi: 10.1146/annurev.micro. 112408.134219

Paget, M. S., Molle, V., Cohen, G., Aharonowitz, Y., and Buttner, M. J. (2001). Defining the disulphide stress response in Streptomyces coelicolor A3(2): identification of the $\sigma$ R regulon. Mol. Microbiol. 42, 1007-1020. doi: 10.1046/ j.1365-2958.2001.02675.x

Perumal, A. S., Vishwakarma, R. K., Hu, Y., Morichaud, Z., and Brodolin, K. (2018). RbpA relaxes promoter selectivity of $M$. tuberculosis RNA polymerase. Nucleic Acids Res. 41, 10106-10118. doi: 10.1093/nar/gky714

Pratt, L. A., and Silhavy, T. J. (1998). Crl stimulates RpoS activity during stationary phase. Mol. Microbiol. 29, 1225-1236. doi: 10.1046/j.1365-2958.1998.01007.x

Rao, X., Deighan, P., Hua, Z., Hu, X., Wang, J., Luo, M., et al. (2009). A regulator from Chlamydia trachomatis modulates the activity of RNA polymerase through direct interaction with the $\beta$ subunit and the primary $\sigma$ subunit. Genes Dev. 23, 1818-1829. doi: 10.1101/gad.1784009

Rodrigue, S., Provvedi, R., Jacques, P., Gaudreau, L., and Manganelli, R. (2006). The $\sigma$ factors of Mycobacterium tuberculosis. FEMS Microbiol. Rev. 30, 926-941. doi: 10.1111/j.1574-6976.2006.00040.x

Saecker, R. M., Record, M. T., and deHaseth, P. L. (2011). Mechanism of bacterial transcription initiation: RNA polymerase - promoter binding, isomerization to initiation-competent open complexes, and initiation of RNA synthesis. J. Mol. Biol. 412, 754-771. doi: 10.1016/j.jmb.2011.01.018 
Schwartz, E. C., Shekhtman, A., Dutta, K., Pratt, M. R., Cowburn, D., Darst, S., et al. (2008). A full-length group 1 bacterial sigma factor adopts a compact structure incompatible with DNA binding. Chem. Biol. 15, 1091-1103. doi: 10.1016/j.chembiol.2008.09.008

Severinovaa, E., Severinova, K., and Darst, S. A. (1998). Inhibition of Escherichia coli RNA polymerase by bacteriophage T4 AsiA. J. Mol. Biol. 279, 9-18. doi: 10.1006/jmbi.1998.1742

Shi, J., Wen, A., Zhao, M., You, L., Zhang, Y., and Feng, Y. (2019). Structural basis of $\sigma$ appropriation. Nucleic Acids Res. 47, 9423-9432. doi: 10.1093/nar/gkz682

Simeonov, M. F., Bieber Urbauer, R. J., Gilmore, J. M., Adelman, K., Brody, E. N., Niedziela-Majka, A., et al. (2003). Characterization of the interactions between the bacteriophage T4 AsiA protein and RNA polymerase. Biochemistry 42, 7717-7726. doi: 10.1021/bi0340797

Tabib-Salazar, A., Liu, B., Doughty, P., Lewis, R. A., Ghosh, S., Parsy, M.-L., et al. (2013). The actinobacterial transcription factor RbpA binds to the principal sigma subunit of RNA polymerase. Nucleic Acids Res. 41, 5679-5691. doi: 10. 1093/nar/gkt277

Tabib-Salazar, A., Mulvenna, N., Severinov, K., Matthews, S. J., and Wigneshweraraj, S. (2019). Xenogeneic regulation of the bacterial transcription machinery. J. Mol. Biol. 431, 4078-4092. doi: 10.1016/j.jmb.2019. 02.008

Tagami, S., Sekine, S., Minakhin, L., Esyunina, D., Akasaka, R., Shirouzu, M., et al. (2014). Structural basis for promoter specificity switching of RNA polymerase by a phage factor. Genes Dev. 28, 521-531. doi: 10.1101/gad.2339 16.113

Vishwakarma, R. K., Cao, A. M., Morichaud, Z., Perumal, A. S., Margeat, E., and Brodolin, K. (2018). Single-molecule analysis reveals the mechanism of transcription activation in M. tuberculosis. Sci. Adv. 4:eaao5498. doi: 10.1126/ sciadv.aao5498

Wu, X., Haakonsen, D. L., Sanderlin, A. G., Liu, Y. J., Shen, L., Zhuang, N., et al. (2018). Structural insights into the unique mechanism of transcription activation by Caulobacter crescentus GcrA. Nucleic Acids Res. 46, 3245-3256. doi: 10.1093/nar/gky161
Xu, J., Cui, K., Shen, L., Shi, J., Li, L., You, L., et al. (2019). Crl activates transcription by stabilizing active conformation of the master stress transcription initiation factor. eLife 8:e50928. doi: 10.7554/eLife.50928

You, L., Shi, J., Shen, L., Li, L., Fang, C., Yu, C., et al. (2019). Structural basis for transcription antitermination at bacterial intrinsic terminator. Nat. Commun. 10:3048. doi: 10.1038/s41467-019-10955-x

Zenkin, N., Kulbachinskiy, A., Yuzenkova, Y., Mustaev, A., Bass, I., Severinov, K., et al. (2007). Region 1.2 of the RNA polymerase $\sigma$ subunit controls recognition of the -10 promoter element. EMBO J. 26, 955-964. doi: 10.1038/sj.emboj. 7601555

Zenkin, N., Severinov, K., and Yuzenkova, Y. (2015). Bacteriophage Xp10 antitermination factor $\mathrm{p} 7$ induces forward translocation by host RNA polymerase. Nucleic Acids Res. 43, 6299-6308. doi: 10.1093/nar/gkv586

Zhang, H., Vellappan, S., Tang, M. M., Bao, X., and Fan, H. (2019). GrgA as a potential target of selective antichlamydials. PLoS One 14:e0212874. doi: 10. 1371/journal.pone.0212874

Zhang, Y., Feng, Y., Chatterjee, S., Tuske, S., Ho, M. X., Arnold, E., et al. (2012). Structural basis of transcription initiation. Science 338, 1076-1080. doi: 10.1126/ science. 1227786

Zuo, Y., and Steitz, T. A. (2015). Crystal structures of the E. coli transcription initiation complexes with a complete bubble. Mol. Cell 58, 534-540. doi: 10. 1016/j.molcel.2015.03.010

Conflict of Interest: The authors declare that the research was conducted in the absence of any commercial or financial relationships that could be construed as a potential conflict of interest.

Copyright (c) 2020 Vishwakarma and Brodolin. This is an open-access article distributed under the terms of the Creative Commons Attribution License (CC BY). The use, distribution or reproduction in other forums is permitted, provided the original author(s) and the copyright owner(s) are credited and that the original publication in this journal is cited, in accordance with accepted academic practice. No use, distribution or reproduction is permitted which does not comply with these terms. 